\title{
Leitthema
}

Gefässchirurgie 2016 $21: 224-231$

DOI 10.1007/s00772-016-0164-4

Online publiziert: 30. Juni 2016

(c) Der/die Autor(en) 2016 . Dieser Artikel ist eine Open-Access-Publikation.

CrossMark

M. Hakimi - M. S. Bischoff · K. Meisenbacher · M. Ante · D. Böckler

Klinik für Gefäßchirurgie und Endovaskuläre Chirurgie, Universitätsklinik Heidelberg, Heidelberg, Deutschland

\section{Der Aortenbogen - was ist bei der endovaskulären Versorgung zu beachten?} gens mit Stentgraftprothesen (TEVAR) hat sich im Verlauf der letzten beiden Jahrzehnte zu einer bewährten Behandlungsoption für entsprechende Läsionen der Aorta entwickelt. Seit der erstmaligen Implantation 1994 [12] wird die TEVAR mittlerweile als erste Option bei den meisten Pathologien eingesetzt [33].

Die offene Rekonstruktion (OR) im Bereich des Aortenbogens stellt nach wie vor den Goldstandard mit einer 30-Tage-
Mortalitätsrate von ca. $2 \%$ dar. Jedoch gelten diese Daten lediglich für ein hochselektioniertes Patientenkollektiv [2, 25, 31].

Die technische Durchführbarkeit von TEVAR im Aortenbogen liegt bei $90 \%$ $[9,37]$ bei einer Mortalität von $0-14 \%$ $[1,27]$. Die Morbidität kann bis zu $55 \%$ betragen, wobei die neurologische Komplikationsrate ca. 5-6\% beträgt. Die Rate an Endolekagen (EL) Typ I und III von etwa $10 \%$ sowie die Mortalität sind von der Höhe der Landungszone (LZ) unabhängig. Die EL-Typ-II-Rate scheint allerdings abhängig von der $\mathrm{LZ}$ zu sein.

Alter $<75$ Jahre, Nierenfunktionsstökuläre Versorgung aufgrund der Indikationsbreite und geringeren Invasivität deutlich häufiger indiziert als die OR [7]. rung und Notfalleingriffe sind dagegen eigenständige prädiktive Faktoren für die Mortalität.
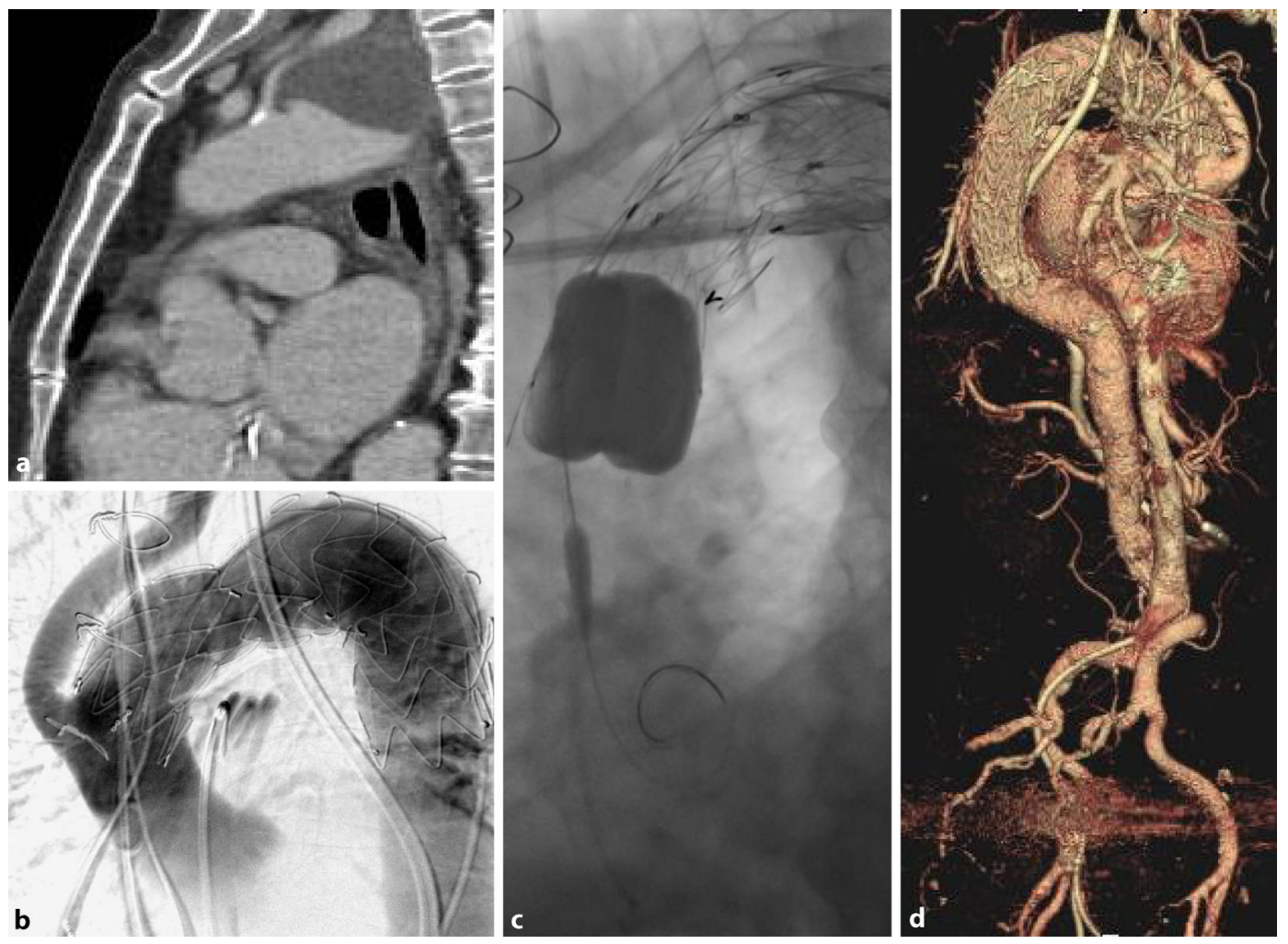

Abb. $1<$ a Endovaskuläre Versorgung eines Aortenbogenaneurysmas, $8 \mathrm{~cm}$ Quer-Diameter.b-d Zur Schaffung einer proximalen Landungszone erfolgt zunächst ein komplettes Debranching der supraaortalen Äste mittels Anlage eines Ascendens-Bypasses auf den Tr. brachiocephalicus, karotidosubklavialem Bypass von re. nach li. mit Reimplantation der linken A. carotis communis sowie proximaler Ligatur der linken A. subclavia (vor dem Abgang der A. vertebralis). Anschließend erfolgt die Implantation eines TEVAR, proximale Landungszone 0 

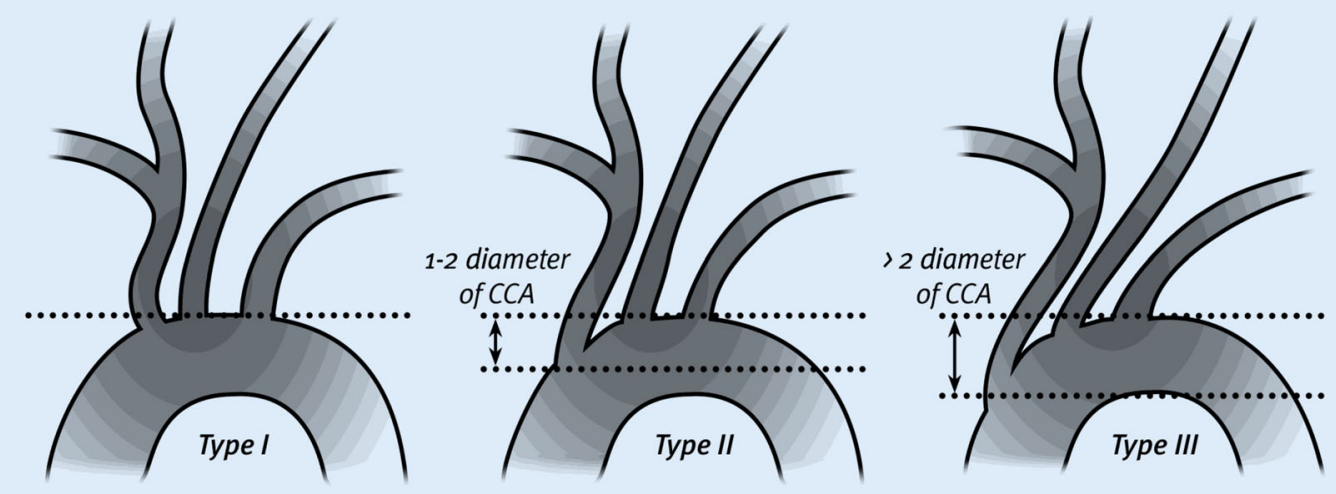

Abb. $2<$ Aortenbogentypen I-III, Typ III (,gothic arch"). (Mit freundl. Genehmigung von: Medienzentrum Universitätsklinikum Heidelberg)
Infobox 1 Einflussfaktoren auf die Apposition und das Alignment der Endoprothese im Bereich der proximalen Landungszone

- Morphologie des Aortenbogens

- Aortenbogentyp,

- Thrombuslast,

- Bemessung der Stentgraftprothese (engl.: "sizing")

- Wahl der Landungszone (Zone 0-4)

- Materialverhalten der Stentgraftprothese

Eine wissenschaftliche Auswertung hinsichtlich des Methodenvergleichs zwischen offenen und endovaskulären Behandlungsoptionen wurde bisher nicht ausreichend durchgeführt. Multizentrische randomisiert-kontrollierte Studien oder Metaanalysen sind nicht vorhanden. Die Datenlage bezieht sich auf Serien einzelner Zentren und Registerstudien [14, 19, 34, 38]. Somit ergibt sich für diese Fragestellung ein Evidenzlevel 3 mit einem Empfehlungsgrad IIa [29, 32].

Besondere Vorteile der TEVAR bestehen bei der Versorgung des akuten Aortensyndroms und Rezidiveingriffen am Aortenbogen [24]. Dementsprechend finden sich in den relevanten Registerstudien bis zu $60 \%$ Patienten mit Notfallindikationen.

Unabhängig von der zu versorgenden Pathologie (thorakales Aortenaneurysma, penetrierendes Aortenulcus, intramurales Hämatom, Aortendissektion, Komplikation von Voroperationen, Transsektion), hängen Resultat und Rate neurologischer Komplikationen der
TEVAR besonders von der adäquaten Wahl der LZ ab [20].

Durch Transposition der supraaortalen Gefäße erweitern sich die Optionen für die Wahl der proximalen LZ bis in die Aorta ascendens (• Abb. 1).

„Snorkel“- oder „Chimney“-Verfahren [26] und Weiterentwicklungen der Stentgraftprothesen mit Implementierung von Fenestrierungen bzw. Verzweigungen („branches“) lassen mittlerweile rein endovaskuläre Prozeduren auch für die LZ 1 und 0 zu. Die Etablierung dieser technischen Neuerungen und die Weiterentwicklung solcher Devices wird eine der Herausforderungen der kommenden Jahre für die endovaskuläre Chirurgie in dieser aortalen Topografie [17].

Gleichwohl an entsprechenden Zentren eine breite Expertise besteht und die TEVAR-Prozedur als Routineverfahren gilt, stellen gerade die Interventionen im Bogenbereich nach wie vor hoch anspruchsvolle Eingriffe dar. Patientenalter und -morbidität (u. a. durch Notfallsituationen aggraviert) bedingen eine relativ hohe Mortalität.

\section{) Patientenalter und -morbidität bedingen eine relativ hohe Mortalität}

Häufig liegt eine komplexe Morphologie bzw. Bogengeometrie vor. Die Position der supraaortalen Äste, Variabilität der Pathologien und die hohe Rate von Notfalleingriffen (Trauma, akute komplizierte Typ-B-Dissektion, Aneurysmaruptur) stellen eine besondere Herausforderung an Indikation und technische Planung sowie Durchführung der Prozedur dar.

Komplikationen aufgrund der anatomischen Topografie wie z. B. zerebrale-, spinale- oder viszerale Ischämie, können erhebliche Folgen haben [36]. Patientenspezifische Gegebenheiten wie z. B. ungünstige Zugangsgefäße, Kinking der Aorta descendens oder abdominalis, Begleiterkrankungen (auch instabile Patienten in der Notfallsituation) oder anatomischen Varianten können zudem erschwerend vorliegen.

Die technischen Anforderungen an die Stentgraftprothesen leiten sich aus den oben genannten Konstellationen ab und sollten für die Variabilität der Anatomien bzw. die Besonderheiten der unterschiedlichen Pathologien geeignet sein.

\section{Stentgraftapposition}

Für die Planung ist eine differenzierte $\mathrm{Ab}$ wägung zwischen technisch übersichtlich gehaltenem Verfahren und optimaler Positionierung der TEVAR-Stentprothese durchzuführen.

Die Apposition und die räumliche Angleichung der Stentgraftprothese an die Aortenwand (engl.: alignment) im Bereich der LZ (v. a. im Bereich der proximalen $\mathrm{LZ}$ ) sind entscheidend für $\mathrm{Ab}$ dichtung und Verankerung sowie für die Ergebnisse im weiteren Verlauf und nehmen bei TEVAR im Aortenbogen eine Schlüsselrolle ein [11].

Zahlreiche Faktoren haben Einfluss auf regelrechte Apposition bzw. Alignment und müssen dementsprechend in Planung und Umsetzung der Pro- 
zedur herausragende Beachtung finden (- Infobox 1).

\section{Morphologie des Aortenbogens}

Die Eigenschaft der proximalen LZ hängt u. a. von der Konfiguration des Aortenbogens ab. Entsprechend der geometrischen Bogenform werden drei Aortenbogentypen unterschieden [8], wobei der Typ 3 den spitzesten Winkel im Scheitelpunkt aufweist und für die TEVAR-Implantation eine große Herausforderung darstellt (• Abb. 2).

Ein steiler Aortenbogen bewirkt ausgeprägte Traktionskräfte auf das Trägersystem der Stentgraftprothese, welche sowohl beim Vorbringen und Positionieren als auch bei deren Freisetzung auf die Prozedur einwirken und z. B. zu einer Fehlpositionierung führen können. Bereits bei der Längenbestimmung der LZ im Bogen als auch der Durchmesserwahl erschwert dieser Bogentyp zudem die Planung. Für die Bestimmung der Länge der proximalen LZ verstärkt sich der ohnehin vorhandene Unterschied der Längenmessung in Innen- bzw. Außenkurvatur des Bogens (• Abb. 3).

Auch die Abschätzung des Verhaltens der Stenprothese hinsichtlich ihrer Adaption an die Geometrie (engl.: conformability) wird erschwert.

Kinkings im Bereich der Beckengefäße oder der Aorta abdominalis bzw. thoracica descendens können den Effekt der Traktionskräfte erheblich verstärken.

Ein weiteres Kriterium zur Beurteilung der Morphologie des Aortenbogens und von relevanter Konsequenz für die Komplikationsraten nach TEVAR ist die Thrombogenität der Aortenbogenwand (- Abb. 4).

Bei Grad-V-Läsionen im Aortenbogen gemäß Feezor et. al. ([15]; • Tab. 1) ist die Schlaganfallrate signifikant erhöht. Etwa $15 \%$ der Patienten zur TEVAR sind von einer derartig fortgeschrittenen Läsion des Aortenbogens betroffen [22].

Schließlich muss bei der Planung auf Varianten der supraaortalen Gefäßversorgung respektive Voroperationen geachtet werden.

Das Vorhandensein einer A. lusoria, eines A.-vertebralis-Abgangs links direkt aus der Aorta oder eines „common-

Gefässchirurgie 2016 21:224-231 DOI 10.1007/s00772-016-0164-4

(C) Der/die Autor(en) 2016. Dieser Artikel ist eine Open-Access-Publikation.

M. Hakimi · M. S. Bischoff · K. Meisenbacher · M. Ante · D. Böckler

\section{Der Aortenbogen - was ist bei der endovaskulären Versorgung zu} beachten?

\section{Zusammenfassung}

Die Versorgung von Aortenpathologien mit Beteiligung des Bogens wird an spezialisierten Zentren mittlerweile vornehmlich endovaskulär durchgeführt. Gemäß der Literatur stellt die offene Rekonstruktion zwar noch den Goldstandard dar, allerdings gilt dies lediglich für ein hochselektioniertes Patientenkollektiv. Dementsprechend ist der Anteil von Versorgungen in Notfallsituationen und bei älteren bzw. vorerkrankten Patienten im TEVAR-Kollektiv besonders hoch. Insgesamt liegt die technische Erfolgsrate der TEVAR im Aortenbogen unabhängig von der Landungszone bei ca. $90 \%$ mit einer Mortalität von 0-14\% und einer Morbidität von bis zu $55 \%$. Durch die anatomische Beziehung zu den supraaortalen Ästen und den diversen Ätiologien mit oft komplexen morphologischen Konfigurationen ist neben der kritischen Indikationsstellung die technische Herangehensweise anspruchsvoll und für den Erfolg maßgebend. Die angemessene Apposition des Stentgrafts im Bereich der proximalen Landungszone ist der wesentliche Aspekt für das klinische Ergebnis. Ein thrombogener Aortenbogen bzw. die Positionierung in Landungszone 1 gehen mit erhöhter neurologischer Morbidität einher. Wird eine Proximalisierung in die Zonen 2-0 notwendig, erfordert dies eine Überstentung der linken A. subclavia bzw. ein Debranching der supraaortalen Gefäße. Durch sog. "Chimney"-Techniken und seit Kurzem eingesetzten fenestrierten bzw. verzweigten ("gebranchten“) TEVAR-Stentgraftprothesen speziell für den Bogen erweitert sich das rein endovaskuläre Repertoire. TEVAR im Aortenbogen ist ein etabliertes Verfahren auch für komplexe Pathologien. Aufgrund schwieriger Indikationsstellung und technischer Komplexität ist aber für die erfolgreiche Umsetzung ein spezialisiertes und zudem erfahrenes Team mit entsprechender Infrastruktur notwendig.

Schlüsselwörter TEVAR · Endovaskuläre Chirurgie · Aorta · Endoleak $\cdot$ Hybridoperation

\section{The aortic arch - what is important for endovascular treatment?}

\section{Abstract}

Aortic arch pathologies are now treated in highly specialized centers and mainly using an endovascular approach. In the literature open surgical repair still represents the gold standard; however, this only applies to a highly selected patient population. Hence patients considered for thoracic endovascular aortic repair (TEVAR) have more comorbidities and are more often treated in emergency situations. For aortic arch pathologies TEVAR is associated with a technical feasibilty rate of $90 \%$, a mortality rate of $0-14 \%$ and a morbidity of up to $55 \%$ independent of the aortic landing zone. Due to the close proximity of the supra-aortic arteries and variable etiologies with highly complex morphological configurations, decisionmaking in favor of aortic repair should be thoroughly considered as the technical approach is challenging but decisive for success. Overall procedural success and long-term results are mainly determined by adequate stent graft deployment in the area of the proximal landing zone. A thrombogenic aortic arch as well as stent graft deployment in landing zone 1 pose a significant risk for neurological morbidities. Landing zones 2-0 require stent graft deployment in the aortic arch subsequently necessitating occlusion of the left subclavian artery and supra-aortic debranching. The so-called chimney techniques and recently developed fenestrated and branched endografts improve the feasibilty of EVAR alone. The use of TEVAR for aortic arch pathologies is a well-established technique even for complex pathologies; however, technical feasibility and success require a highly specialized and experienced team as well as technical equipment in order to handle challenging aortic pathologies.

Keywords TEVAR - Endovascular surgery - Aorta · Endoleak . Hybrid procedure 

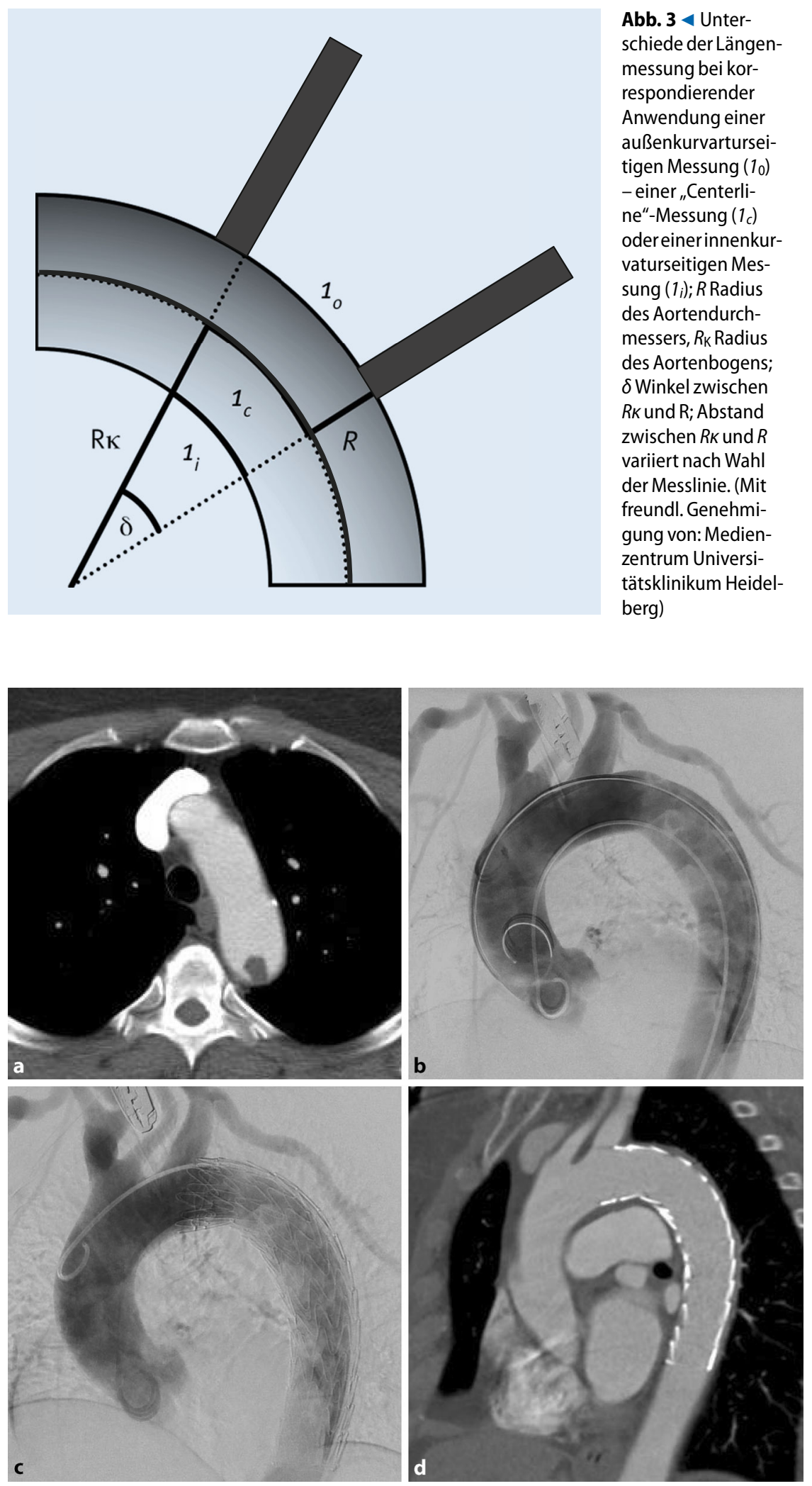

Abb. $4 \Delta$ a Computertomographische Angiographie eines flottierenden Thrombus im Aortenbogen vor Planung zur TEVAR. Angiografie vor (b) und nach (c) Freisetzen der Prothese sowie gutes Alignment bei Absetzen in Landungszone 3 (d) ostiums" der A. carotis communis und der A. subclavia (LSA) links können ebenso wie z. B. ein A.-mammaria-Bypass die üblicherweise vorhandene LZ beeinträchtigen. Vor allem in Notfallsituationen ist die korrekte Befundung durch Zeitnot, Verdrängung der Anatomie aufgrund einer expansiven Dissektion bzw. Aneurysmaformation oder eines Hämatoms relevant erschwert.

\section{Wahl der proximalen Landungszone}

Es gibt wenig Evidenz hinsichtlich des Zusammenhangs zwischen der Wahl der LZ und der Morbidität und Mortalität.

Die Höhe der proximalen LZ (d.h. prox. LZ in Zone 0-4) selber stellt keinen Risikofaktor für die Überlebensrate dar.

Vor allem die Morbidität bezüglich neurologischer Ereignisse hängt (wie o. g.) von der thrombogenen Morphologie des Aortenbogens im Bereich der proximalen LZ sowie der Absetzung in LZ 1 ab [20].

Eine Proximalisierung der LZ-Höhe (Zone 0-1) ging in publizierten Fallserien mit Zunahme der EL-Rate einher [16, 21,27]. Dies könnte Folge einer Abnahme der Apposition sein [27], da bei proximaler Absetzung und v. a. steilen Aortenbogenkonfigurationen ausgeprägte Kräfte auf die Stengraftprothese wirken. Die Innenkurvatur wirkt als Hebelpunkt auf die Prothese, die zudem ausgeprägten pulsatilen Bewegungskräften des Bogens unterliegt.

In der Literatur bzw. den Leitlinien ist keine minimale Länge für die LZ vorgegeben. Die Herstellerangaben (IFU - engl.: „instructions for use“) der kommerziell erhältlichen TEVAR-Devices variieren von 15-25 mm [10, 18]. Die Querdurchmesser gängiger Stentgraftprodukte variieren von $16-24 \mathrm{~mm}$ im minimalen und $38-46 \mathrm{~mm}$ im maximalen Prothesendurchmesser.

\section{Bogenhybrideingriffe}

Für das Auftreten perioperativer und verlaufsbedingter Komplikationen sind die Qualität und die Länge der LZ insbesondere der proximalen LZ, wesentliche Faktoren. Nach Mitchell u. Ishimaru wird 
Tab. 1 Einteilung der Thrombogenität des Aortenbogens nach Feezor et al. [15]

\section{Atheromlast des Aortenbogens}

\begin{tabular}{|ll|}
\hline Grad I & Normal \\
\hline Grad II & Intimaverdickung \\
\hline Grad III & Atherom $<5 \mathrm{~mm}$ \\
\hline Grad IV & Atherom $\geq 5 \mathrm{~mm}$ \\
\hline Grad V & $\begin{array}{l}\text { Ulzeriertes, zerklüftetes Atherom } \\
\text { („mobile lesion“) }\end{array}$ \\
\hline
\end{tabular}

die Lokalisation der LZ in Zone 0-4 unterteilt ([28]; und • Abb. 5).

Eine Proximalisierung der LZ nach Zone 2 bzw. bis Zone 0 ermöglicht die Realisation einer ausreichenden Länge der LZ, birgt allerdings weiterführende Überlegungen oder Operationsschritte, da die Perfusion der supraaortalen Äste mittels Revaskularisationsverfahren zumindest bei Positionierung in LZ 1 und 0 gewährleistet werden muss.

Bei Positionierung in Zone 2 ist eine Abwägung hinsichtlich Indikation und Zeitpunkt einer Revaskularisation der linken A. subclavia (LSA) erforderlich.

Folge der Überstentung können Durchblutungsstörungen des linken Arms, Subclavian-Steal-Phänomen/ Syndrom, Typ II EL, spinale Ischämie oder Ischämie im A.-vertebralis-Versorgungsgebiet sein.

Strenge Kriterien für eine Revaskularisation sind ein A.-mammaria-Bypass auf Koronararterien, ein ipsilateraler Dialyse-AV-Shunt, der Verschluss der rechten A. vertebralis und Varianten der supraortalen Abgänge wie z. B. eine A. lusoria oder ein isolierter Abgang der A. vertebralis li. oder ein linksseitiger Tr. brachiocephalicus. Relative Gründe sind die Situation bei dominanter linker Hand, insbesondere bei manueller beruflicher Tätigkeit. Vorangegangene aortale Operationen begünstigen das Auftreten einer spinalen Ischämie, da die Gesamtlänge der prothetal versorgten Aorta das Risiko erhöht $[3,20]$.

Die Revaskularisation mittels Transposition oder karotidosubklavialem Bypass gewährleistet die Perfusion der LSA bei TEVAR in Zone 2. Allerdings gehen mit diesem Operationsschritt eigene relevante Risiken einher. Die Schlaganfallrate bzw. die Gesamtmorbidität der A.-subclavia-Revaskularisation übersteigt den

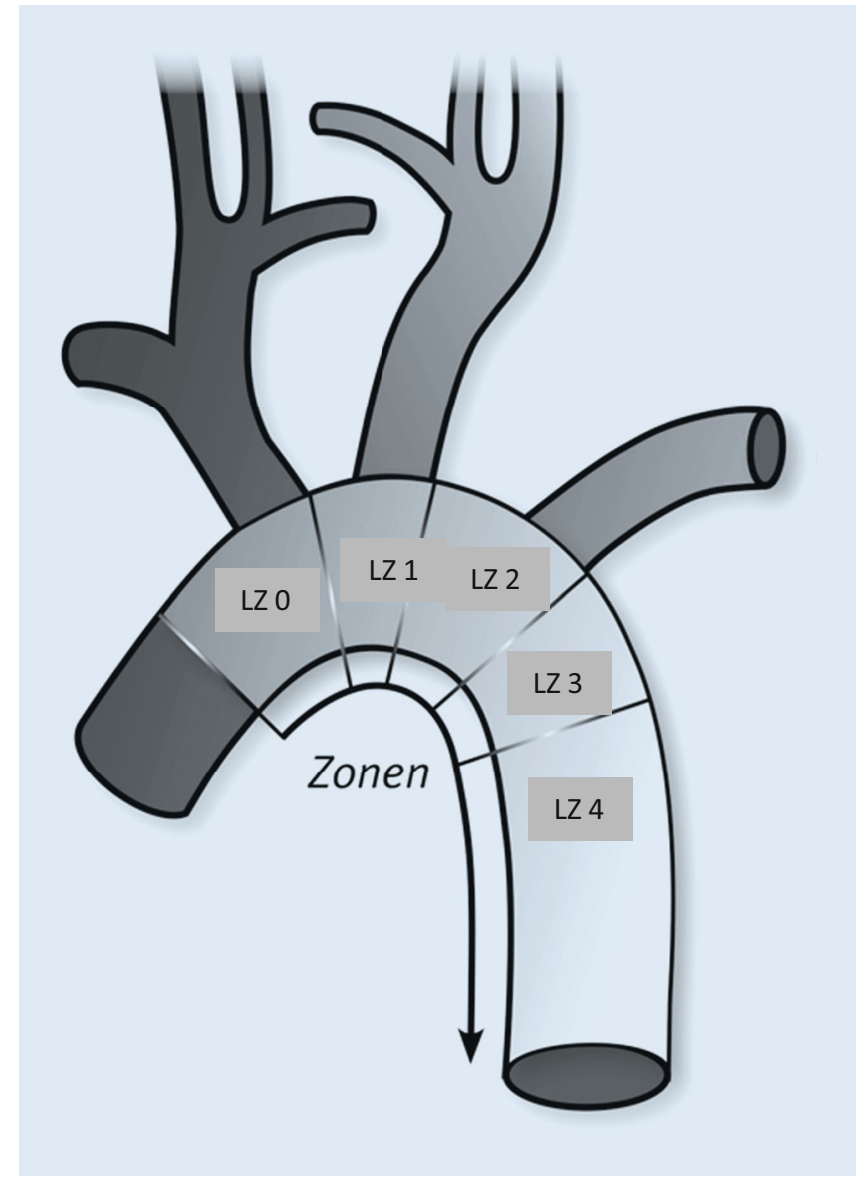

Abb. $5<$ Landungszonen für TEVAR im Aortenbogen. (Nach [29]. Mit freundl. Genehmigung von: Medienzentrum Universitätsklinikum Heidelberg)

Vorteil einer regulär gestellten bzw. prophylaktischen Indikation [20].

Für die Positionierung der TEVAR in Zone 2 kann somit festgehalten werden, dass die Revaskularisation der LSA ein partikuläres Komplikationsrisiko birgt und daher in ausgewählten Konstellationen einzeitig, ansonsten selektiv zweizeitig im Fall des Auftretens von Symptomen erfolgen sollte.

Die Positionierung in LZ 1 erfordert ein sog. Semi-Debranching, die Implantation eines karotidosubklavialen Bypasses von rechts nach links mit Reinsertion der linken A. carotis communis und geht mit dem statistisch höchsten Schlaganfallrisiko einher. Die Indikation zur Positionierung der TEVAR in dieser LZ kann als eigenständiger Risikofaktor für Komplikationen bei TEVAR angesehen werden [22].

Wie herausfordernd die TEVAR als Bogen-Hybrideingriff ist, zeigt sich daran, dass etwa $20 \%$ aller Patienten einen Hybrideingriff mit TEVAR in den Zonen 0 bis 2 benötigen, bei einer Morta- litätsrate von ca. 19\% [21]. Dabei ist ein weiterer Anstieg der Mortalität bei komplettem Debranching sowie bei Notfalloperationen zu sehen [5].

\section{Technische Kriterien der Prothese}

Die anatomischen Verhältnisse im Bereich des Aortenbogens stellen hohe Ansprüche an die Funktion der Stentgraftprothese. Die Apposition sollte außenund innenkurvarturseitig gleichermaßen gegeben sein. Hierfür ist eine ausreichende Anpassung an die äußerst variable Form des Aortenbogens erforderlich. Die radiale Kraft sollte eine ausreichende $\mathrm{Ab}$ dichtung und Fixierung gewährleisten, ohne selber zu Wanddefekten zu führen [13], was insbesondere bei Dissektionen und intramuralen Hämatomen bzw. traumatischen Läsionen gravierende Folgen haben könnte. Dies wird bei den neusten TEVAR-Device-Generationen durch eine adaptierende radiale Kraft erreicht, die zudem die „Sizing“-Breite erhöht. 

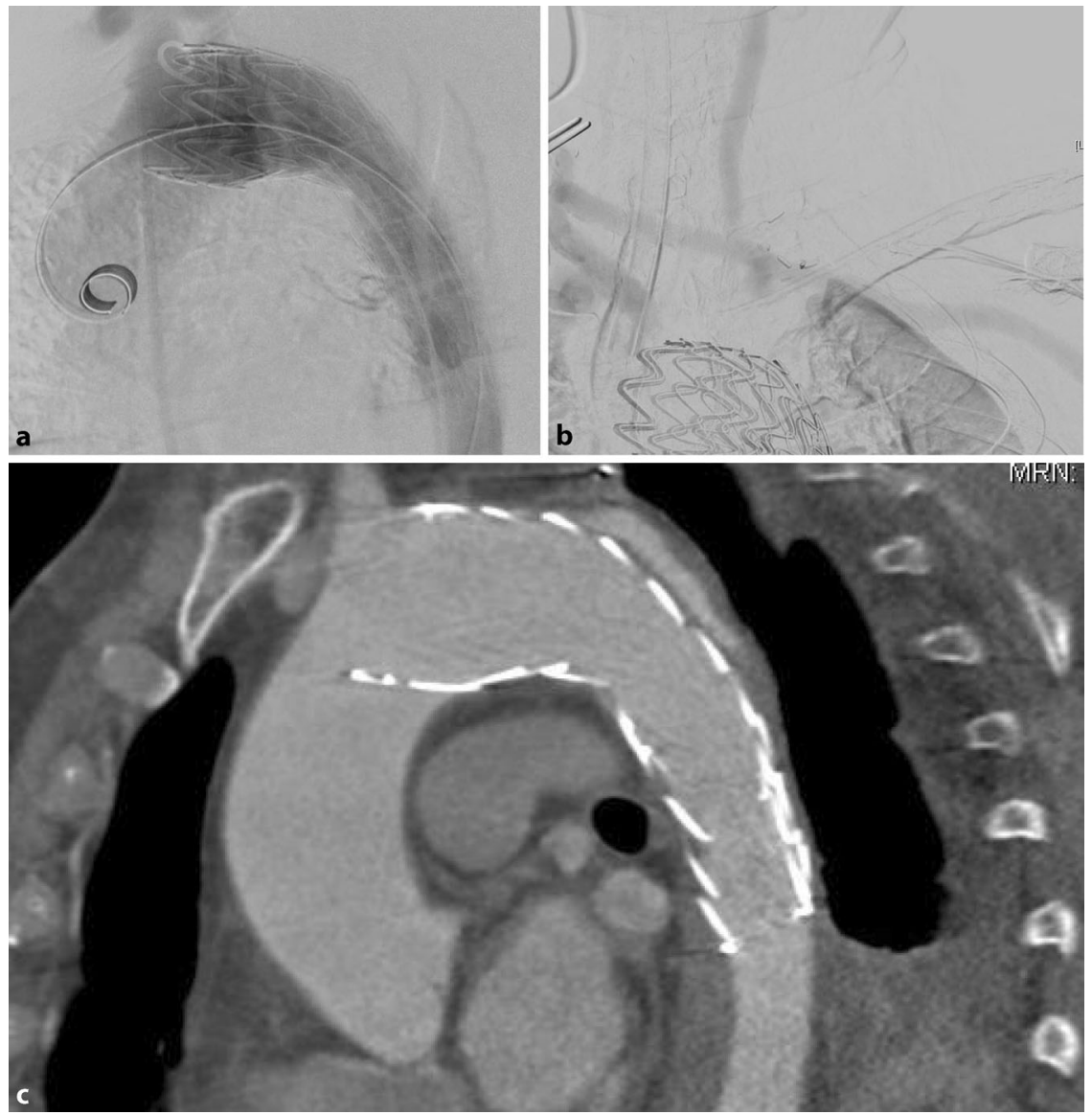

Abb. $6 \Delta$ a Absetzung in Landungszone 1.b Semi-Debranching beiÜberstenten der A. carotis communis links. c Ausgeprägtes „bird's beaksign“ bei erschwerter Anpassungsfähigkeit in Zone 1 als proximale Landungszone

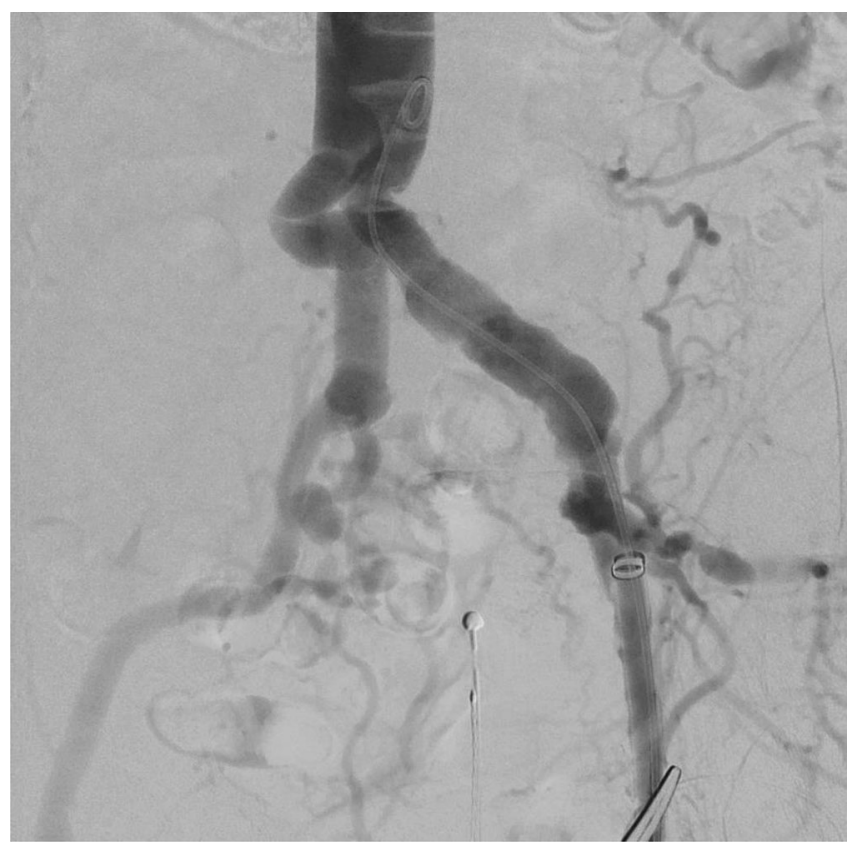

Abb. $7<$ SchwierigerZugangsweg bei Z.n. aortobiilakaler Y-Prothese (Dacron) vor Jahren. Elongation und Kinking der Prothesenschenkel
Diese und ausreichende Durchmesservariationen bzw. konische Durchmesservarianten (engl.: tapered) erhöhen die Einsetzbarkeitsbreite und verringern bei bestimmten morphologischen Varianten den Einsatz mehrerer Stentgraftprothesen.

Der Freisetzungsmechanismus spielt eine entscheidende Rolle bei der Positionierung, aber auch bei der Konformabilität der Prothese. Stentgraftprothesen mit in der Mitte beginnender Freisetzung können an Hebelpunkten frühzeitig Kontakt zur Aortenwand aufnehmen und entweder fehlpositioniert werden oder eine ungünstige Verteilung der zurückfedernden Kräfte herbeiführen, was die Apposition ungünstig beeinflussen kann (v. a.im Bereich der Innenkurvatur) [6]. Die dabei entstandene Inkongruenz des Prothesenverlaufs zur Aortengeometrie, in der Literatur „bird's beak“ (• Abb. 6) genannt erhöht die Wahrscheinlichkeit technischer Komplikationen wie z. B. Stentgraftkollaps oder Endoleak [35].

Die technischen Applikationen der neuen Stentgraftgeneration zeigen daher erhöhte Konformabilität durch Flexibilität, adaptive radiale und rückfedernde Kräfte (in der longitudinalen Achse wirkende Kräfte, die von der Prothese auf die Aortenwand übertragen werden und ebenso auf die Längsausrichtung der Prothese wirken).

Ein weiteres Kriterium zur Performance der Devices ist das Durchmesserprofil des Trägersystems. Sogenannte „Low-profile“-Trägersysteme ermöglichen eine genauere Steuerbarkeit der Prothese entlang der gesamten iliakoaortalen Strecke, was besonders bei ausgeprägtem „Kinking“ ein entscheidender Aspekt bei der erfolgreichen Implantation sein kann.

Auch die bei der Ausmessung der Längen- und Durchmesserverhältnisse zur Prothesenwahl angewendeten Kriterien sind strittig. Einige Hersteller geben in ihren IFU die Ausmessung anhand einer außenkurvarturseitigen Längenmessung, andere anhand eine "Centerline“ an. Abb. 3 verdeutlicht die Unterschiede, die bzgl. Apposition und Länge der LZ gemessen werden können. 

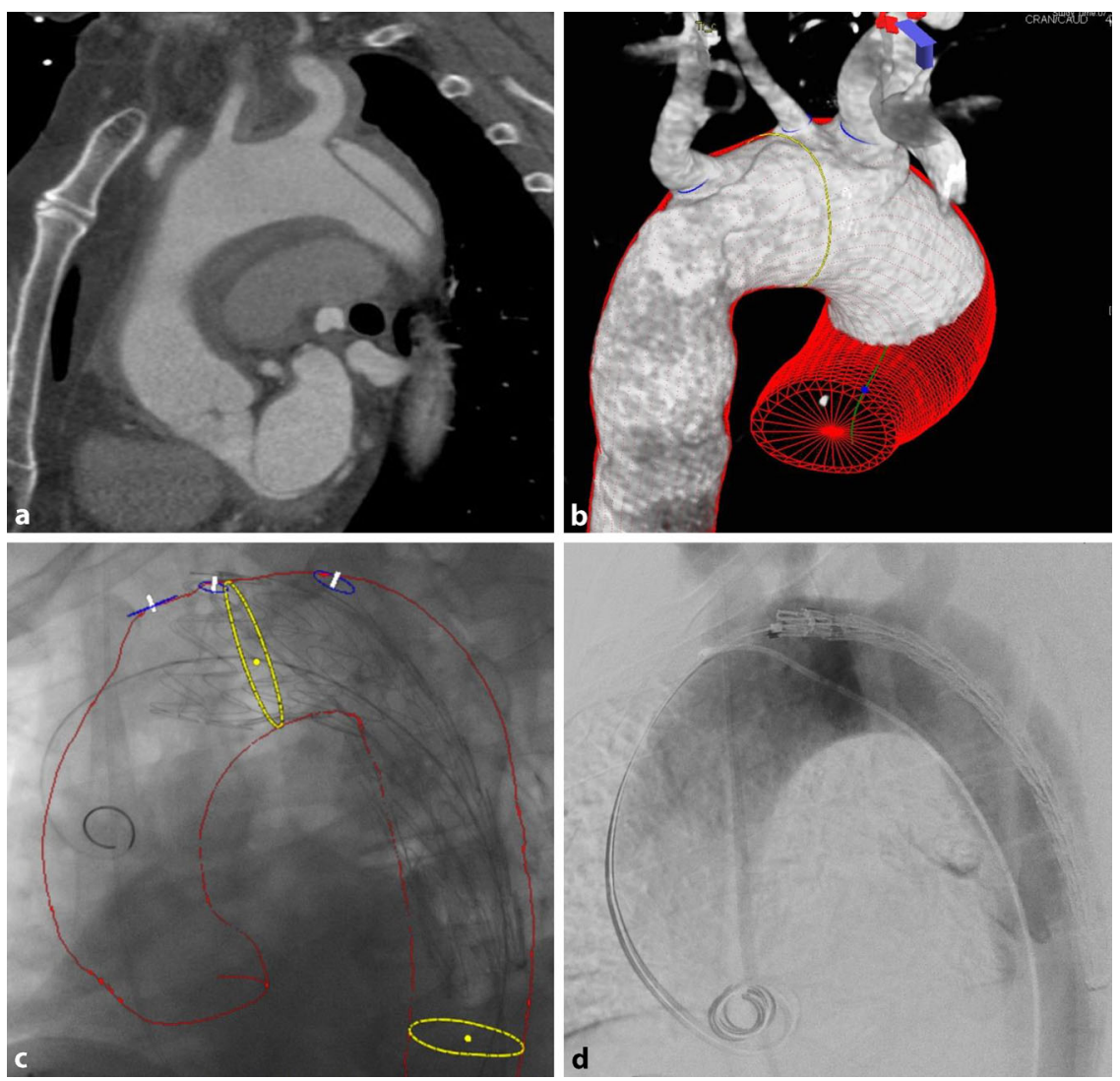

Abb. $8 \Delta$ a Endovaskuläre Versorgung einer Aortendissektion Typ Stanford B mit Entry direkt ab Abgang der linken A. subclavia). b-d Intraoperative Planung und Platzierung der Prothese in Landungszone 2

\section{Branched Devices}

Aktuell stehen sechs Stentgraftprothesen für die endovaskuläre Versorgung im Aortenbogen mit Fenestrierungen bzw. Verzweigungen (,branches") zur Verfügung. Die Weiterentwicklungen der endovaskulären Technologie in dieser Gefäßregion weisen technisch unterschiedliche Spezifikationen auf und sind bisher in limitierter Konstellation zur Anwendung gekommen. Die aktuellen Publikationen präliminärer Ergebnisse zeigen eine erfolgreiche Umsetzbarkeit in einem selektionierten Patientenkollektiv [17].

\section{Allgemeine Kriterien}

Zusätzlich sind weitere Faktoren für die Durchführbarkeit und die erfolgreiche TEVAR-Implantation maßgebend. Die Durchmesser, die Kalzifikation und die Morphologie (Kinking) der Beckenarterien können limitierende Ursachen für einen transfemoralen Zugang sein. Hier wird ein Zugang über die proximalen Beckengefäße (A. iliaca communis) oder die Aorta abdominalis erforderlich, für den z. B. ein übergangsweiser Bypass als Zugangsweg (sog. Conduit) mit einer alloplastischen Prothese hilfreich sein kann [4].

Voroperationen bzw. Kinkings der Aorta selbst können ebenfalls limitierende Faktoren für das intraluminale Vorbringen und Platzieren der Prothese sein (• Abb. 7).

Der intraoperativen Bildgebung kommt eine wichtige Bedeutung zu, insbesondere wenn die gewählte Länge der proximalen LZ kurz ist (außerhalb der IFU). Zur intraoperativen Darstellung der LZ ist daher die Parallaxenkorrektur auf den Winkel des distalen supraaortalen Gefäßes (je nach LZ: LSA oder li. ACC) einzustellen. Bei komplexen Geometrien kann dieses kompliziert werden und ist in modernen Hybrid-Operationssälen wohl am genausten einstellbar.
In einem solchen Setting kann auch Fusionsbildgebung oder intraoperative Navigation ( $\bullet$ Abb. 8) eingesetzt werden und ein weiteres unterstützendes Instrument sein [30].

Weiterhin sind Aspekte der interdisziplinären Planung mit der Anästhesie wie „rapid pacing“ zur exakten Prothesenfreisetzung oder Liquordrainage im Sinne der Risikominimierung einer spinalen Ischämie von Bedeutung [23].

\section{Fazit für die Praxis}

- Für ein selektioniertes Patientengut stellt die offene Rekonstruktion den Goldstandard dar.

- Der Evidenzgrad ist bei fehlenden multizentrischen RCT oder Metaanalysen gering.

- Im derzeitigen klinischen Alltag werden die meisten Fälle endovaskulär versorgt.

- TEVAR zeigt für die diversen Pathologien (TAA, PAU, IMH, AD) eine vergleichbare Ergebnisqualität.

- Eine hohe Thrombogenität des Aortenbogens und die Positionierung in LZ 1 sind Risikofaktoren für neurologische Komplikationen nach TEVAR.

- Hybridoperationen und "Chimney"Technik erweitern das Repertoire.

- Dissektionen stellen eine besondere Herausforderung dar.

- Konformabilität und Anpassung der radialen Kräfte der Stentgraftprothese gewährleistet die erfolgreiche Positionierung mit guter Apposition.

- Qualität und Wahl der proximalen LZ sind der Schlüssel zur erfolgreichen TEVAR-Prozedur im Aortenbogen.

- Dabei scheint die Qualität hinsichtlich Winkel und Thrombogenität im Bereich der proximalen LZ wichtiger als die reine Länge zu sein (bei niedrigem Evidenzlevel).

- Akkurate Messung, Bildgebung und Kenntnisse der Prothesenmechanik sind besonders während der Implantation wichtig. 


\section{Korrespondenzadresse}

PD Dr. med. M. Hakimi
Klinik für Gefäßchirurgie und
Endovaskuläre Chirurgie,
Universitätsklinik Heidelberg
Im Neuenheimer Feld 110,
$\begin{aligned} & 69120 \text { Heidelberg, } \\ & \text { Deutschland } \\ & \text { Maani.Hakimi@med.uni- } \\ & \text { heidelberg.de }\end{aligned}$

\section{Einhaltung ethischer Richtlinien}

Interessenkonflikt. M. Hakimi, M. S. Bischoff, K. Meisenbacher, M. Ante und D. Böckler geben an, dass kein Interessenkonflikt besteht.

Dieser Beitrag beinhaltet keine von den Autoren durchgeführten Studien an Menschen oder Tieren.

Open Access. Dieser Artikel wird unter der Creative Commons Namensnennung 4.0 International Lizenz (http://creativecommons.org/licenses/by/4.0/deed. de) veröffentlicht, welche die Nutzung, Vervielfältigung, Bearbeitung, Verbreitung und Wiedergabe in jeglichem Medium und Format erlaubt, sofern Sie den/die ursprünglichen Autor(en) und die Quelle ordnungsgemäßnennen, einen Linkzur Creative Commons Lizenz beifügen und angeben, ob Änderungen vorgenommen wurden.

\section{Literatur}

1. Bergeron $P$, Coulon $P$, De Chaumaray $T$ et al (2005) Great vessels transposition and aortic arch exclusion. J Cardiovasc Surg (Torino) 46:141-147

2. BischoffMS, Brenner RM, Scheumann J et al (2010) Long-term outcome after aortic arch replacement with a trifurcated graft. J Thorac Cardiovasc Surg 140:S71-S76

3. Bischoff MS, Peters AS, Kotelis D et al (2011) Strategische Überlegungen bei mit spinaler Ischämie assoziertem intramuralem Hämatom. Gefässchirurgie 16:346-349

4. Bischoff MS, Peters AS, Meisenbacher $K$ et al (2014) Challenging access in endovascular repair of infrarenal aortic aneurysms. J Cardiovasc Surg (Torino) 55:75-83

5. Bischoff MS, Ante M, Meisenbacher $K$ et al (2016) Outcome of thoracic endovascular aortic repair in patients with thoracic and thoracoabdominal aortic aneurysms. J Vasc Surg. doi:10.1016/j.jvs.2015.11.045

6. Böckler D, Müller-Eschner M, Tengg-Kobligk Het al (2014) Conformability in Aortic Type B Dissection. Endovasc Today Suppl:8-11

7. Buth J, Harris PL, Hobo R et al (2007) Neurologic complications associated with endovascular repair of thoracic aortic pathology: Incidence and risk factors.astudy from the EuropeanCollaborators on Stent/Graft Techniques for Aortic Aneurysm Repair (EUROSTAR) registry. J Vasc Surg 46:1103-1111

8. Casserly IP, Kapadia SR (2005) Advances in percutaneous valvular intervention. Expert Rev Cardiovasc Ther 3:143-158
9. Chan YC, Cheng SW, Ting AC et al (2008) Supra-aortic hybrid endovascular procedures for complex thoracic aortic disease: single center early to midterm results. JVasc Surg 48:571-579

10. Coady MA, Ikonomidis JS, Cheung AT et al (2010) Surgical management of descending thoracic aortic disease:open and endovascularapproaches: a scientific statement from the American Heart Association. Circulation 121:2780-2804

11. Czerny M, Gottardi R, Zimpfer D et al (2007) Mid-term results of supraaortic transpositions for extended endovascular repair of aortic arch pathologies. Eur J Cardiothorac Surg 31:623-627

12. Dake MD, Miller DC, Semba CP et al (1994) Transluminal placement of endovascular stentgrafts for the treatment of descending thoracic aortic aneurysms. N Engl J Med 331:1729-1734

13. Eggebrecht $H$, Thompson $M$, Rousseau $H$ et al (2009) Retrograde ascending aortic dissection during or after thoracic aortic stent graft placement: insight from the European registry on endovascular aortic repair complications. Circulation 120:S276-281

14. Farber MA, Giglia JS, Starnes BW et al (2013) Evaluation of the redesigned conformable GORE TAG thoracic endoprosthesis for traumatic aortic transection. J Vasc Surg 58:651-658

15. Feezor RJ, Martin TD, Hess PJ et al (2007) Risk factors for perioperative stroke during thoracic endovascular aortic repairs (TEVAR). J Endovasc Ther 14:568-573

16. Gottardi R, Funovics M, Eggers N et al (2008) Supraaortic transposition for combined vascular and endovascular repair of aortic arch pathology. Ann Thorac Surg 86:1524-1529

17. Haulon S, Greenberg RK, Spear Ret al (2014) Global experience with an inner branched arch endograft. JThorac Cardiovasc Surg 148:1709-1716

18. Hiratzka LF, Bakris $G L$, Beckman JA et al (2010) 2010 ACCF/AHA/AATS/ACR/ASA/SCA/ SCAI/SIR/STS/SVM guidelines for the diagnosis and management of patients with thoracic aortic disease: a report of the American College of Cardiology Foundation/American Heart Association Task Force on Practice Guidelines, American Association for Thoracic Surgery, American College of Radiology, American Stroke Association, Society of Cardiovascular Anesthesiologists, Society for Cardiovascular Angiography and Interventions, Society of Interventional Radiology, Society of Thoracic Surgeons, and Society for Vascular Medicine. Circulation 121:e266-e369

19. Jordan WD Jr., Rovin J, Moainie Setal (2015) Results of a prospective multicenter trial of CTAG thoracic endograft. JVasc Surg 61:589-595

20. Kotelis D, Geisbusch P, Hinz U et al (2009) Short and midterm results after left subclavian artery coverage during endovascular repair of the thoracic aorta. JVasc Surg 50:1285-1292

21. Kotelis D, Geisbusch P, Attigah N et al (2011) Total vs hemi-aortic arch transposition for hybrid aortic arch repair. JVasc Surg 54:1182-1186

22. Kotelis D, Bischoff MS, Jobst B et al (2012) Morphological risk factors of stroke during thoracic endovascular aortic repair. Langenbecks Arch Surg 397:1267-1273

23. Kotelis D, Bianchini C, Kovacs B et al (2015) Early experience with automatic pressure-controlled cerebrospinal fluid drainage during thoracic endovascular aortic repair. J Endovasc Ther 22:368-372

24. Kotelis D, Bischoff MS, Rengier F et al (2016) Endovascular repair of pseudoaneurysms after open surgery for aortic coarctation. Interact Cardiovasc Thorac Surg 22:26-31

25. Lemaire SA, Price MD, Parenti JL et al (2011) Early outcomes after aortic arch replacement by using the Y-graft technique. Ann Thorac Surg 91:700-708

26. Mangialardi N, RoncheyS, Malaj A etal (2015) Value and limitations of chimney grafts to treat arch lesions. J Cardiovasc Surg (Torino) 56:503-511

27. Melissano G, Civilini E, Bertoglio L et al (2007) Results of endografting of the aortic arch in different landing zones. Eur J Vasc Endovasc Surg 33:561-566

28. Mitchell RS, Ishimaru S, Ehrlich MP et al (2002) First International Summit on Thoracic Aortic Endografting: roundtable on thoracic aortic dissection as an indication for endografting. JEndovasc Ther 9(Suppl 2):I|98-II105

29. Mitchell RS, Ishimaru S, Criado FJ et al (2005) Third international summit on thoracic aortic endografting: lessons from long-term results of thoracic stent-graft repairs. J Endovasc Ther 12:89-97

30. Schulz CJ, Schmitt M, Bockler D et al (2016) Feasibility and accuracy of fusion imaging during thoracic endovascular aortic repair. J Vasc Surg 63:314-322

31. SettepaniF, Cappai A, Basciu A etal (2016) Outcome of open total arch replacement in the modern era. JVasc Surg 63:537-545

32. Stather PW, Sayers RD, Cheah A et al (2012) Outcomes of endovascular aneurysm repair in patients with hostile neck anatomy. Eur J Vasc Endovasc Surg 44:556-561

33. Svensson LG, Kouchoukos NT, Miller DC et al (2008) Expert consensus document on the treatment of descending thoracic aortic disease using endovascular stent-grafts. Ann Thorac Surg 85:S1-S41

34. Torsello GB, Torsello GF, Osada N et al (2010) Midterm results from the TRAVIATA registry: treatment of thoracic aortic disease with the valiant stent graft. JEndovasc Ther 17:137-150

35. Ueda T, Fleischmann D, Dake MD et al (2010) Incomplete endograft apposition to the aortic arch: bird-beak configuration increases risk of endoleak formation after thoracic endovascular aortic repair. Radiology 255:645-652

36. Ullery BW, Mcgarvey M, Cheung AT et al (2012) Vascular distribution of stroke and its relationship toperioperative mortality and neurologicoutcome after thoracic endovascular aortic repair. J Vasc Surg 56:1510-1517

37. Zhou W, Reardon M, Peden EK et al (2006) Hybrid approach to complex thoracic aortic aneurysms in high-risk patients: surgical challenges and clinical outcomes. JVasc Surg 44:688-693

38. Zipfel B, Chiesa R, Kahlberg A et al (2014) Endovascular repair of traumatic thoracic aortic injury: final results from the relay endovascular registry for thoracic disease. Ann Thorac Surg 97:774-780 